\title{
Maximal pseudocompact spaces and the Preiss-Simon property
}

\author{
Ofelia T. Alas ${ }^{1 *}$, Vladimir V. Tkachuk ${ }^{2 \dagger}$, Richard G. Wilson ${ }^{2 \neq}$
}

1 Instituto de Matemática e Estatística, Universidade de São Paulo, Caixa Postal 66281, 05311-970 São Paulo, Brasil

2 Departamento de Matemáticas, Universidad Autónoma Metropolitana, Av. San Rafael Atlixco, 186, Col. Vicentina, Iztapalapa, C.P. 09340, Mexico D.F., Mexico

Received 8 March 2013 ; accepted 13 July 2013

Abstract: We study maximal pseudocompact spaces calling them also MP-spaces. We show that the product of a maximal pseudocompact space and a countable compact space is maximal pseudocompact. If $X$ is hereditarily maximal pseudocompact then $X \times Y$ is hereditarily maximal pseudocompact for any first countable compact space $Y$. It turns out that hereditary maximal pseudocompactness coincides with the Preiss-Simon property in countably compact spaces. In compact spaces, hereditary MP-property is invariant under continuous images while this is not true for the class of countably compact spaces. We prove that every Fréchet-Urysohn compact space is homeomorphic to a retract of a compact MP-space. We also give a ZFC example of a Fréchet-Urysohn compact space which is not maximal pseudocompact. Therefore maximal pseudocompactness is not preserved by continuous images in the class of compact spaces.

MSC: 54D99, 54B10

Keywords: Maximal pseudocompact space $\cdot$ Countably compact space $\cdot$ MP-space $\cdot$ Compact space $\cdot$ Pseudocompact space $\cdot$ Preiss-Simon property

(C) Versita Sp. z o.o.

\section{Introduction}

A topological space $X$ (with no separation axioms assumed) is called feebly compact if every locally finite family of nonempty open subsets of $X$ is finite. Maximal feebly compact spaces were studied in $[4,12,13]$. In [3] the maximality of this

\footnotetext{
*E-mail: alas@ime.usp.br

+ E-mail:vova@xanum.uam.mx

* E-mail: rgw@xanum.uam.mx
} 
class was studied in the realm of Tychonoff spaces. To avoid confusions, we will follow the terminology of [3] calling a space pseudocompact if it is feebly compact and Tychonoff. Accordingly, a space $X$ is said to be maximal pseudocompact or an MP-space if it is pseudocompact and any stronger Tychonoff topology on $X$ fails to be pseudocompact.

It is easy to see that any first countable pseudocompact space is maximal pseudocompact; it was asked in [3, Question 2.24] whether Fréchet-Urysohn property in compact spaces is sufficient to imply maximal pseudocompactness. In this paper we give an example showing that a Fréchet-Urysohn compact space need not be maximal pseudocompact. It is still an open problem to find an internal characterization of maximal pseudocompactness.

Maximal pseudocompactness is not preserved by products even in compact spaces: the relevant example was given in [3]. On the other hand, it was proved in [3] that $X \times(\omega+1)$ is maximal pseudocompact whenever $X$ is maximal pseudocompact We generalize this result establishing that $X \times M$ is maximal pseudocompact if $X$ is maximal pseudocompact and $M$ is a countable compact space.

We call a space $X$ hereditarily maximal pseudocompact if every closed subspace of $X$ is maximal pseudocompact. It turns out that hereditary MP-property is closely related to the Preiss-Simon property introduced and studied in functional analysis and $C_{p}$-theory. Recall that $X$ is a Preiss-Simon space or has the Preiss-Simon property if for every closed set $F \subset X$, each point $x \in F$ is a limit of a sequence of non-empty open subsets of $F$. We will establish that a countably compact space $X$ is hereditarily maximal pseudocompact if and only if it is a Preiss-Simon space. We also prove that the Preiss-Simon property is multiplicative in the products of countably compact spaces with compact first countable spaces. As a consequence, for any hereditarily maximal pseudocompact space $X$, the space $X \times Y$ has to be hereditarily maximal pseudocompact for any first countable compact $Y$.

We also show that every Fréchet-Urysohn compact space is a retract of compact Fréchet-Urysohn maximal pseudocompact space. As an easy consequence, maximal pseudocompactness is not preserved by continuous images in compact spaces. On the other hand, the hereditary maximal pseudocompactness is invariant under continuous maps in the class of compact spaces but not in countably compact spaces.

\section{Notation and terminology}

All spaces are assumed to be Tychonoff. If $X$ is a space then $\tau(X)$ is its topology and $\tau^{*}(X)=\tau(X) \backslash\{\varnothing\}$. Given any point $x \in X$ let $\tau(x, X)=\{U \in \tau(X): x \in U\}$. The set $\mathbb{R}$ is the real line with its usual topology and $\mathbb{D}=\{0,1\}$ is the doubleton with the discrete topology.

A sequence $\left\{A_{n}: n \in \omega\right\}$ of subsets of a space $X$ is said to converge to a point $x \in X$ if for any $U \in \tau(x, X)$ there exists $m \in \omega$ such that $A_{n} \subset U$ for all $n \geq m$. A space $X$ has the Preiss-Simon property if for every closed set $F \subset X$ and every $x \in F$, there exists a sequence of non-empty open subsets of $F$ that converges to $x$. Given a space $X$, a set $U \in \tau(X)$ is called regular open if $U=\operatorname{lnt} \bar{U}$; a set $F \subset X$ is regular closed if $F=\bar{V}$ for some open $V \subset X$. A space $X$ is called semiregular if regular open sets form a base in $X$.

For any $X$, the tightness $t(X)$ of the space $X$ is the minimal cardinal $\kappa$ such that $\bar{A}=\bigcup\{\bar{B}: B \subset A$ and $|B| \leq \kappa\}$ for any $A \subset X$. A space $X$ is Fréchet-Urysohn, if for any $A \subset X$ and $x \in \bar{A}$, there is a sequence $\left\{a_{n}: n \in \omega\right\} \subset A$ with $a_{n} \rightarrow x$. A compact space $X$ is called Eberlein compact if $X$ embeds into a space $\Sigma_{*}(\kappa)=\left\{x \in \mathbb{R}^{\kappa}\right.$ : for any $\varepsilon>0$ the set $\{\alpha<\kappa:|x(\alpha)|>\varepsilon\}$ is finite $\}$ for some cardinal $\kappa$. If for some cardinal $\kappa$, a compact space $X$ embeds in the space $\Sigma(\kappa)=\left\{x \in \mathbb{R}^{\kappa}:|\{\alpha<\kappa: x(\alpha) \neq 0\}| \leq \omega\right\}$, then $X$ is called Corson compact.

If $X$ is a space and $x \in X$ then a family $\mathcal{B} \subset \tau^{*}(X)$ is called a local $\pi$-base at $x$ if every $U \in \tau(x, X)$ contains an element of $\mathcal{B}$. The minimal cardinality of a local $\pi$-base at $x$ is called $\pi$-character of $X$ at $x$ and is denoted by $\pi \chi(x, X)$. The $\pi$-character $\pi \chi(X)$ of the space $X$ is the cardinal $\sup \{\pi \chi(x, X): x \in X\}$.

The rest of our notation is standard and follows the book [6]. 


\section{Maximal pseudocompactness in subspaces and products}

It was proved in the paper [3] that the product of two hereditarily maximal pseudocompact spaces need not be maximal pseudocompact. On the other hand, if $X$ is maximal pseudocompact then $X \times(\omega+1)$ is also maximal pseudocompact: this was also proved in [3]. We will generalize this result and give a sufficient condition for a product of two hereditarily maximal pseudocompact spaces to be hereditarily maximal pseudocompact.

[3, Theorem 2.4] implies that any Fréchet-Urysohn pseudocompact space with a dense set of isolated points is maximal pseudocompact. In particular, the one-point compactification of a discrete space is maximal pseudocompact; this shows that the cardinality of a maximal pseudocompact space is unbounded.

\section{Proposition 3.1.}

If $X$ is a maximal pseudocompact space then $\pi \chi(X) \leq \omega$ and $|X| \leq d(X)^{\omega}$.

Proof. If $x$ is an isolated point of $X$ then clearly $\pi \chi(x, X) \leq \omega$. If $x \in X$ is not isolated then by [3, Theorem 2.1] there exists a sequence $\mathcal{S}=\left\{U_{n}: n \in \omega\right\}$ of non-empty open subsets of $X$ which converges to $x$. It is evident that $\mathcal{S}$ is a countable $\pi$-base at $x$ so $\pi \chi(x, X) \leq \omega$. To prove the second inequality, take a dense set $D \subset X$ such that $|D|=d(X)$ and observe that every point of the space $X$ is a limit of a sequence from $D$ (see [3, Lemma 2.8]). Therefore $|X| \leq|D|^{\omega}=d(X)^{\omega}$.

\section{Corollary 3.2.}

Any maximal pseudocompact paratopological group is compact and metrizable.

Proof. If $G$ is a maximal pseudocompact paratopological group then $G$ is a topological group by [14, Theorem 2.6]; we have $\pi \chi(G) \leq \omega$ by Proposition 3.1. It follows from [2, Proposition 1.1] that $\chi(G) \leq \omega$ so $G$ is metrizable and hence compact.

\section{Proposition 3.3.}

Suppose that $X$ is a space and we can find a non-clopen set $A \subset X$ such that both sets $A$ and $X \backslash A$ are pseudocompact. Then $X$ is not maximal pseudocompact.

Proof. The space $A \oplus(X \backslash A)$ has the same underlying set $X$ and a strictly stronger pseudocompact topology.

\section{Corollary 3.4.}

If a space $X$ has a proper dense pseudocompact subspace then $X$ is not maximal pseudocompact.

Proof. If $Y$ is a dense pseudocompact subspace of $X$ and $Y \neq X$ then fix a point $x \in X \backslash Y$ and observe that $X \backslash\{x\}$ is also pseudocompact because $Y$ is dense in $X \backslash\{x\}$. Therefore $\{X \backslash\{x\},\{x\}\}$ is a partition of $X$ into pseudocompact non-clopen subsets so $X$ is not an MP-space by Proposition 3.3.

\section{Corollary 3.5.}

If $X$ is a pseudocompact non-compact space then no compactification of $X$ is maximal pseudocompact.

\section{Proposition 3.6.}

A space $X$ is compact and maximal pseudocompact if and only if $\beta X$ is maximal pseudocompact.

Proof. If $X$ is compact and maximal pseudocompact then $\beta X=X$ is maximal pseudocompact. Now, if $\beta X$ is maximal pseudocompact and $X$ is not compact then take a point $p \in \beta X \backslash X$ and observe that $\beta(\beta X \backslash\{p\})=\beta X$ so we can apply $[8,6]]$ to conclude that $\beta X \backslash\{p\}$ is pseudocompact. Therefore we have a decomposition $\{\beta X \backslash\{p\},\{p\}\}$ of the space $\beta X$ into two disjoint pseudocompact non-clopen subsets so $\beta X$ is not MP by Proposition 3.3 which is a contradiction. 
Recall that a compact space $X$ is called dyadic if $X$ is a continuous image of $\mathbb{D}^{\kappa}$ for some cardinal $\kappa$.

Theorem 3.7.

A dyadic space $X$ is maximal pseudocompact if and only if $X$ is metrizable.

Proof. Every metrizable compact space is maximal pseudocompact so it suffices to prove necessity. Assume that $X$ is an MP-space and fix a continuous onto map $\varphi: \mathbb{D}^{k} \rightarrow X$; consider the $\Sigma$-product $S=\left\{x \in \mathbb{D}^{k}:\left|x^{-1}(1)\right| \leq \omega\right\}$ of the space $\mathbb{D}^{k}$. It is well known (and easy to see) that $S$ is a dense countably compact subset of $\mathbb{D}^{k}$. If $\varphi(S)=X$ then the space $X$ is a continuous image of $S$ so it is metrizable by [5, Theorem 10]. If $Y=\varphi(S) \neq X$ then $Y$ is a proper dense countably compact subspace of $X$ so $X$ is not an MP-space by Corollary 3.4 which is a contradiction.

\section{Theorem 3.8.}

The following conditions are equivalent for any linearly ordered space $X$ :

(a) $X$ is maximal pseudocompact;

(b) $X$ is hereditarily maximal pseudocompact;

(c) $X$ is countably compact and first countable.

Proof. It is straightforward that $(\mathrm{c}) \Rightarrow(\mathrm{b}) \Rightarrow(\mathrm{a})$. To prove $(\mathrm{a}) \Rightarrow(\mathrm{c})$ assume that $(X,<)$ is an MP-space. Since any linearly ordered space is normal, $X$ has to be countably compact. It $t(X)>\omega$ then fix a set $A \subset X$ and a point $x \in X$ such that $x \in \bar{A}$ but $x \notin \bar{B}$ for any countable $B \subset A$. There is no loss of generality to consider that $A \subset L_{x}=\{y \in X: y<x\}$. Since $A$ is cofinal in $L_{x}$, for any countable set $E \subset L_{x}$ we can find a countable $B \subset A$ such that for any $x \in E$ there is $b \in B$ with $x \leq b$. It follows from $x \notin \bar{B}$ that there exists $y<x$ such that $(y, x] \cap B=\emptyset$ and hence $(y, x] \cap E=\emptyset$.

As an immediate consequence, $\bar{E} \subset L_{x}$ for any countable $E \subset L_{x}$ which shows that $L_{x}$ is countably compact. Therefore $\left\{L_{X}, X \backslash L_{x}\right\}$ is a decomposition of $X$ into disjoint non-clopen countably compact subsets so $X$ is not maximal pseudocompact by Proposition 3.3, a contradiction. Therefore $X(X)=t(X) \leq \omega$ (see $[6,3.12 .4(\mathrm{~d})])$.

The following theorem shows that the condition in Proposition 3.3 is not equivalent to a space being not maximal pseudocompact.

\section{Theorem 3.9.}

There exists a compact space $X$ that fails to be maximal pseudocompact while for every subset $A \neq \emptyset$ of the space $X$ which is not clopen, either $A$ or $X \backslash A$ is not pseudocompact.

Proof. Let $K=\left(\omega_{1} \times[0,1)\right) \cup\left\{\left(\omega_{1}, 0\right)\right\}$ and consider the topology on $K$ generated by the lexicographic order. The space $K$ is compact; it is usually called the long segment. The space $L=K \backslash\left\{\left(\omega_{1}, 0\right)\right\}$ is called the long line; it is easy to see that it is countably compact and first countable.

Let $p=(0,0)$ and $q=\left(\omega_{1}, 0\right)$; then $p$ is the minimal point of $K$ and $q$ is its maximal point. Denote by $X$ the quotient space obtained by collapsing the set $\{p, q\}$ to a point $u$. Let $\varphi(x)=x$ for any $x \in L \backslash\{p\}$ and $\varphi(p)=u$. Then $\varphi: L \rightarrow X$ is a continuous bijection which is not a homeomorphism because the inverse map is not continuous at the point $u$. Therefore the space $X$ is not maximal pseudocompact.

The space $X$ is first countable at any point $x \in X \backslash\{u\}$ and hence we have the following property:

$$
\text { for any } x \in X \text { and any pseudocompact set } A \subset X \text {, if } x \in \bar{A} \backslash A \text { then } x=u \text {. }
$$

Assume that $A$ and $B$ are non-empty disjoint pseudocompact subsets of $X$ such that $A \cup B=X$. If $A \backslash\{u\} \neq \emptyset$ and $B \backslash\{u\} \neq \emptyset$ then fix points $a \in A \backslash\{u\}$ and $b \in B \backslash\{u\}$; there is no loss of generality to consider that $a<b$. It follows from property (1) that $A^{\prime}=A \cap[a, b]$ and $B^{\prime}=B \cap[a, b]$ are closed subsets of $[a, b]=\{x \in X: a \leq x \leq b\}$. Therefore $[a, b]$ is the union of two disjoint closed subsets which contradicts connectedness of $[a, b]$. Therefore one of the sets $A, B$ is equal to $\{u\}$ and the other is $X \backslash\{u\}$. However, $X \backslash\{u\}$ is not pseudocompact because letting $U_{n}=\{0\} \times\left(2^{-n-1}, 2^{-n}\right)$ for any $n \in \omega$ we obtain a sequence $\left\{U_{n}: n \in \omega\right\}$ of non-empty open subsets of $X$ that converges to $u$. 
Theorem 3.10.

If $X$ is a maximal pseudocompact space and $M$ is a countable compact space then $X \times M$ is maximal pseudocompact.

Proof. Every countable compact space $M$ is homeomorphic to a countable ordinal $\alpha+1$ with its order topology (see [10]). It was proved in [3] that $X \times(\omega+1)$ is maximal pseudocompact for any maximal pseudocompact space $X$.

Suppose that $\alpha \in \omega_{1}$ is the smallest ordinal such that $X \times(\alpha+1)$ is not maximal pseudocompact; note that $\alpha$ is necessarily a limit ordinal, for otherwise, $X \times(\alpha+1)$ is a finite union of clopen maximal pseudocompact spaces and so is maximal pseudocompact. Let $\tau$ be the product topology on $Y=X \times(\alpha+1)$ and let $v$ be the topology of $X$. Since $Y$ is not maximal pseudocompact, there is a strictly stronger pseudocompact topology $\sigma$ on $Y$. We are assuming that $\alpha$ is the minimal ordinal for which $X \times(\alpha+1)$ is not maximal pseudocompact so $\tau$ and $\sigma$ coincide on $X \times(\beta+1)$ for each $\beta<\alpha$ and hence they also coincide on $X \times \alpha$. Given any set $E \subset Y$ and $\beta \leq \alpha$ let $E_{\beta}=\{x \in X:(x, \beta) \in E\}$.

Claim 1. For any point $z$ in the space $(X, v)$ if $(z, \alpha) \in A \in \sigma$ then there exists $\gamma<\alpha$ such that $z \in \mathrm{cl}_{v}\left(A_{\beta}\right)$ for every $\beta>\gamma$.

Proof. Fix a set $A \in \sigma$; if $z$ is isolated in $(X, v)$ then the set $F=\{z\} \times(\alpha+1)$ is clopen in $(Y, \tau)$ and hence in $(Y, \sigma)$ so it is a pseudocompact subset of $(Y, \sigma)$ and hence the restrictions of $\tau$ and $\sigma$ on $F$ coincide. This implies that $A$ is a $\tau$-neighbourhood of $(z, \alpha)$ so our claim is trivially true.

Now, if $z$ is not isolated in $X$ then it follows from maximal pseudocompactness of $(X, v)$ that the point $z$ is the limit of a sequence $\mathcal{T}=\left\{B_{n}: n \in \omega\right\} \subset v \backslash\{\emptyset\}$ (see [3, Theorem 2.1]). It turns out that the set

$$
J=\left\{\beta \in \alpha: \text { there are infinitely many } m \in \omega \text { such that } B_{m} \not \subset A_{\beta}\right\}
$$

cannot be cofinal in $\alpha$. To see this, assume the contrary; then we can find a set $I=\left\{\beta_{n}: n \in \omega\right\} \subset J$ such that $\beta_{n}<\beta_{n+1}$ for each $n \in \omega$ and $I$ is cofinal in $\alpha$. It takes an easy recursion to choose a strictly increasing sequence $\left\{n_{j}: j \in \omega\right\} \subset \omega$ such that $B_{n_{j}} \not \subset A_{\beta_{j}}$ for every $j \in \omega$. The sequence $\left\{B_{n_{j}}: j \in \omega\right\} \subset \mathcal{T}$ still converges to $z$. Now, for each $j \in \omega$ let $\delta_{j}=\beta_{j}-1$ if $\beta_{j}$ is a successor ordinal; if $\beta_{j}$ is a limit ordinal then pick any ordinal $\delta_{j}$ such that $\beta_{j-1}<\delta_{j}<\beta_{j}$.

The family $\mathcal{B}=\left\{B_{n_{j}} \times\left(\delta_{j}, \beta_{j}\right]: j \in \omega\right\}$ consists of $\tau$-open (and hence $\sigma$-open) subsets of $Y$. Since $(Y, \sigma)$ is pseudocompact, $\mathcal{B}$ must have an accumulation point; we will show that this can only be $(z, \alpha)$.

To prove this, note first that if $\beta<\alpha$ then for any $x \in X$ the set $X \times(\beta+1)$ is a neighbourhood of the point $(x, \beta)$ which meets only finitely many elements of $\mathcal{B}$. On the other hand if $x \neq z$, then since $\left\{B_{n_{j}}: j \in \omega\right\}$ must also converge to $z$, there is a neighbourhood $U$ of $x$ which meets only finitely many elements of the family $\left\{B_{n_{j}}: j \in \omega\right\}$. But then, $U \times(\alpha+1)$ meets only finitely many elements of $\mathcal{B}$. Therefore the family $\mathcal{B}$ converges to $(z, \alpha)$ in $(Y, \tau)$ and hence also in $(Y, \sigma)$, since this latter space is pseudocompact; thus there is $k \in \omega$ such that whenever $n_{j} \geq k$, we have $B_{n_{j}} \times\left(\delta_{j}, \beta_{j}\right] \subset A$ and so $B_{n_{j}} \subset A_{\beta_{j}}$, a contradiction.

This shows that for some $\gamma \in \alpha$ and all $\beta>\gamma$, the set $A_{\beta}$ contains all but finitely many elements of $\mathcal{T}$. It follows that $z \in \operatorname{cl}_{v}\left(A_{\beta}\right)$ for all $\beta>\gamma$, i.e., Claim 1 is proved.

Claim 2. For any $x \in X$, if a set $I \subset \alpha$ is cofinal in $\alpha$ and $\{(x, \beta): \beta \in I\} \subset A \in \sigma$ then $(x, \alpha) \in \operatorname{cl}_{\sigma}(A)$.

Proof. If $p=(x, \alpha) \notin \mathrm{cl}_{\sigma}(A)$ then $U=Y \backslash \mathrm{cl}_{\sigma}(A)$ is a $\sigma$-neighbourhood of $p$ so we can use Claim 1 to find an ordinal $\gamma<\alpha$ such that $x \in \mathrm{cl}_{v}\left(U_{\beta}\right)$ for any $\beta>\gamma$. In particular, there exists $\beta>\gamma$ with $\beta \in$ I, i.e., $(x, \beta) \in A=\operatorname{Int}_{\sigma}(A)$; it follows from $x \in \mathrm{cl}_{\nu}\left(U_{\beta}\right)$ that the point $(x, \beta)$ belongs to $\tau$-closure of $U$ and hence $(x, \beta) \in \mathrm{cl}_{\sigma}(U)$ because the topologies $\tau$ and $\sigma$ coincide at $(x, \beta)$. This contradiction shows that $p \in \mathrm{cl}_{\sigma}(A)$, i.e., Claim 2 is proved.

Take any set $A \in \sigma \backslash \tau$; then there exists a point $(z, \alpha) \in A_{\alpha}$ such that $A$ is not a $\tau$-neighbourhood of $(z, \alpha)$ and hence $(z, \alpha) \in \operatorname{Bd}_{\tau}(A) \cap A$. Since $(Y, \sigma)$ is a Tychonoff space, there are $\sigma$-open sets $U, V$ and $W$ such that

$$
(z, \alpha) \in U \subset \mathrm{cl}_{\sigma}(U) \subset V \subset \mathrm{cl}_{\sigma}(V) \subset W \subset \mathrm{cl}_{\sigma}(W) \subset A,
$$

and $W$ is a regular open set (so $Y \backslash W$ is a regular closed set). 
Since $z \in U_{\alpha}$, it follows from Claim 1 that there is some $\beta_{0} \in \alpha$ such that $z \in \mathrm{cl}_{v}\left(U_{\beta}\right) \subset V_{\beta}$ for all $\beta>\beta_{0}$. Without loss of generality, we assume that this holds for all $\beta \in \alpha$.

Let $J=\left\{\mu_{n}: n \in \omega\right\}$ be a cofinal subset of $\alpha$ such that $\mu_{n}<\mu_{n+1}$ for each $n \in \omega$. Since every $V_{\mu_{n}}$ belongs to $v$ and contains $z$, we can find a sequence $\left\{R_{n}: n \in \omega\right\} \subset v$ such that $z \in R_{n}$ and $\mathrm{cl}_{v}\left(R_{n+1}\right) \subset R_{n} \subset V_{\mu_{0}} \cap \cdots \cap V_{\mu_{n}}$ for all $n \in \omega$. Letting $S_{n}=R_{n} \times\left(\mu_{n}, \alpha\right]$ for every $n \in \omega$ we obtain a sequence $\left\{S_{n}: n \in \omega\right\}$ of $\tau$-open neighbourhoods of $(z, \alpha)$. Observe that $\mathrm{cl}_{\sigma}\left(S_{k}\right) \subset \mathrm{cl}_{\tau}\left(S_{k}\right)=\mathrm{cl}_{v}\left(R_{k}\right) \times\left[\mu_{k}, \alpha\right]$ for all $k \in \omega$ and therefore

$$
\bigcap\left\{\mathrm{cl}_{\sigma}\left(S_{k}\right): k \in \omega\right\} \subset \bigcap\left\{\mathrm{cl}_{v}\left(R_{k}\right): k \in \omega\right\} \times\{\alpha\}=\bigcap\left\{R_{k}: k \in \omega\right\} \times\{\alpha\} .
$$

Now, if $x \in \bigcap\left\{R_{k}: k \in \omega\right\}$, then $x \in V_{\mu_{k}}$ and so $\left(x, \mu_{k}\right) \in V$ for each $k \in \omega$; by Claim 2, we have $(x, \alpha) \in \mathrm{cl}_{\sigma}(V) \subset W$. This implies that

$$
\bigcap\left\{\mathrm{cl}_{\sigma}\left(S_{k}\right): k \in \omega\right\} \subset \bigcap\left\{\mathrm{cl}_{v}\left(R_{k}\right): k \in \omega\right\} \times\{\alpha\} \subset W .
$$

Now if for each $k \in \omega$, the set $\mathrm{cl}_{\sigma}\left(S_{k}\right)$ meets $Y \backslash W$, then the family $\left\{\mathrm{cl}_{\sigma}\left(S_{k}\right): k \in \omega\right\} \cup\{Y \backslash W\}$ would be a centered family of regular closed sets with empty intersection. Since the space $(Y, \sigma)$ is pseudocompact, there is $m \in \omega$ such that $\mathrm{cl}_{\sigma}\left(S_{m}\right) \subset W \subset A$, i.e., $S_{m}$ is a $\tau$-neighbourhood of $(z, \alpha)$ contained in $A$, contradicting the hypothesis that $A$ is not a $\tau$-neighbourhood of $(z, \alpha)$.

\section{Theorem 3.11.}

If $X$ is maximal pseudocompact then $X \times \omega_{1}$ is maximal pseudocompact.

Proof. If $X \times \omega_{1}$ is not maximal pseudocompact, then there is a pseudocompact topology $\sigma$ on $X \times \omega_{1}$ strictly stronger than the product topology $\tau$. The topologies $\tau$ and $\sigma$ must differ at some point $(x, \alpha) \in X \times \omega_{1}$ and so $\sigma \mid(X \times(\alpha+1))$ is a pseudocompact topology on $X \times(\alpha+1)$ stronger than $\tau \mid(X \times(\alpha+1))$, showing that $X \times(\alpha+1)$ is not maximal pseudocompact which is a contradiction with Theorem 3.10 .

We will see later that hereditary maximal pseudocompactness behaves better with respect to products because it is equivalent to a convergence property.

\section{Theorem 3.12.}

For a space $X$, the following are equivalent:

(a) every pseudocompact subset of $X$ is maximal pseudocompact;

(b) every pseudocompact closed subset of $X$ is maximal pseudocompact;

(c) every pseudocompact subspace of $X$ is closed;

(d) if $F$ is a pseudocompact closed subspace of $X$ then every point $x \in F$ is a limit of a sequence of non-empty open subsets of $F$.

Proof. The implication (a) $\Rightarrow$ (b) being trivial, assume that (b) holds and suppose that $A \subset X$ is pseudocompact but not closed; then $\mathrm{cl}(A)$ is a closed pseudocompact subspace of $X$ which is not maximal pseudocompact because it has a proper pseudocompact subset $A$ (see Corollary 3.4). This proves (b) $\Rightarrow$ (c).

(c) $\Rightarrow$ (d) Take any closed pseudocompact set $F \subset X$. If $x$ is an isolated point of $F$ then $x$ is a limit of a sequence of non-empty open sets coinciding with $\{x\}$. If $x$ is not isolated then $F \backslash\{x\}$ is not closed and hence it is not pseudocompact. If $\mathcal{U}=\left\{U_{n}: n \in \omega\right\}$ is a discrete family of non-empty open subsets of $F \backslash\{x\}$ then $\mathcal{U}$ converges to $x$.

(d) $\Rightarrow$ (c) Assume that (d) holds while $A \subset X$ is pseudocompact and not closed; fix a point $p \in \bar{A} \backslash A$. Then $\bar{A} \backslash\{p\}$ is pseudocompact because $A$ is its dense pseudocompact subspace. Take a sequence $\left\{U_{n}: n \in \omega\right\}$ of non-empty open subsets of $\bar{A}$ which converges to $p$. It is straightforward that $\left\{U_{n} \backslash\{p\}: n \in \omega\right\}$ is a discrete family of non-empty open subsets of $\bar{A} \backslash\{p\}$ so $\bar{A} \backslash\{p\}$ is not pseudocompact, a contradiction.

(c) $\Rightarrow$ (a) Assume that there is a pseudocompact subspace $F \subset X$ which is not maximal pseudocompact. Let $\sigma$ be a pseudocompact topology on $F$ which is strictly stronger than $\tau \mid F$. Since both $\tau \mid F$ and $\sigma$ are Tychonoff (hence semiregular) topologies, there is a regular closed set $C$ in $(F, \sigma)$ which is not closed in $(F, \tau \mid F)$. The space $(C, \sigma)$ being pseudocompact, the set $C$ is a non-closed pseudocompact subspace of $(X, \tau)$. 


\section{Corollary 3.13.}

For a countably compact space $X$ the following are equivalent:

(a) $X$ is hereditarily maximal pseudocompact;

(b) every pseudocompact subspace of $X$ is closed;

(c) $X$ has the Preiss-Simon property.

In an Eberlein compact space, every pseudocompact subspace is closed (see [11, Corollary 6]), hence the next result follows immediately.

\section{Corollary 3.14.}

Each Eberlein compact space is hereditarily maximal pseudocompact.

The following example answers [3, Question 2.24].

\section{Example 3.15.}

There exists a compact space $X$ such that $C_{p}(X)$ is $K$-analytic (i.e., $X$ is Talagrand compact) while the space $X$ is not maximal pseudocompact. In particular, $X$ must have the Fréchet-Urysohn property being Corson compact.

Proof. Reznichenko constructed a compact space $X$ for which $C_{p}(X)$ is $K$-analytic and there exists a point $x \in X$ such that $X=\beta(X \backslash\{x\})$ and therefore $X \backslash\{x\}$ is pseudocompact (see [8,6]]). This example seems to have never been published; however, it was described (with a complete proof) in $[7, \S 8.4]$. Finally, apply Corollary 3.4 to see that $X$ is not maximal pseudocompact because $X \backslash\{x\}$ is a proper dense pseudocompact subspace of $X$.

\section{Theorem 3.16.}

Suppose that $X$ is a Preiss-Simon space and $f: X \rightarrow Y$ is a closed continuous onto map such that for any closed set $G \subset Y$ there exists a closed $F \subset X$ such that $f(F)=G$ and $f\lceil F: F \rightarrow G$ is irreducible. Then $Y$ is a Preiss-Simon space.

Proof. Suppose that $G \subset Y$ is closed and $y$ is a non-isolated point of $G$. We can find a closed set $F \subset X$ such that $f(F)=G$ and $f\lceil F: F \rightarrow G$ is irreducible; pick a point $x \in F$ such that $f(x)=y$. Since $X$ has the PreissSimon property, $x$ is the limit of a sequence $\left\{U_{n}: n \in \omega\right\}$ of open subsets of $F \backslash\{x\}$. It is straightforward that the sequence $\left\{f\left(U_{n}\right): n \in \omega\right\}$ converges to $y$ and hence so does the sequence $\mathcal{S}=\left\{f^{\sharp}\left(U_{n}\right): n \in \omega\right\}$ where as usual, $f^{\#}\left(U_{n}\right)=G \backslash f\left(F \backslash U_{n}\right)$ for every $n \in \omega$. Since every set $f^{\#}\left(U_{n}\right)$ is non-empty and open in $G \backslash\{y\}$, the sequence $\mathcal{S}$ witnesses that $Y$ has the Preiss-Simon property.

\section{Corollary 3.17.}

If $X$ is a Preiss-Simon space, then every perfect image of $X$ is a Preiss-Simon space.

Proof. Suppose that $f: X \rightarrow Y$ is a perfect map; if $G \subset Y$ is closed then the map $f\left\lceil f^{-1}(G): f^{-1}(G) \rightarrow G\right.$ is also perfect so there exists a closed set $F \subset f^{-1}(G)$ such that $f(F)=G$ and $f\lceil F: F \rightarrow G$ is irreducible. Therefore Theorem 3.16 is applicable to conclude that $Y$ is a Preiss-Simon space.

\section{Corollary 3.18.}

If $X$ is a paracompact Preiss-Simon space and $f: X \rightarrow Y$ is a continuous closed onto map, then $Y$ is also a Preiss-Simon space. 
Proof. If $G \subset Y$ is closed then $f^{-1}(G)$ is a paracompact space and the map $f\left\lceil f^{-1}(G): f^{-1}(G) \rightarrow G\right.$ is also closed. The space $X$ has the Fréchet-Urysohn property which is preserved by closed maps; therefore $G$ is a Fréchet-Urysohn space. This makes it possible to apply $\left[9\right.$, Lemma 5.4] to see that there exists a closed set $F \subset f^{-1}(G)$ such that $f(F)=G$ and $f\lceil F: F \rightarrow G$ is irreducible. Therefore we can apply Theorem 3.16 again to convince ourselves that $Y$ has the Preiss-Simon property.

\section{Corollary 3.19.}

Every continuous image of a compact hereditarily maximal pseudocompact space is hereditarily maximal pseudocompact.

\section{Corollary 3.20.}

Every continuous image of a first countable compact space is hereditarily maximal pseudocompact.

\section{Remark 3.21.}

If $p \notin \omega_{1}$ then the space $X=\omega_{1} \oplus\{p\}$ is hereditarily maximal pseudocompact being countably compact and first countable. It was noted in [3] that $X$ maps continuously onto the space $\omega_{1}+1$ which is not maximal pseudocompact. Therefore compactness in Corollary 3.19 cannot be omitted.

\section{Theorem 3.22.}

If $X$ is a Fréchet-Urysohn compact space, then there exists a compact Fréchet-Urysohn MP-space $K$ such that $X$ is a retract of $K$.

Proof. If $X$ is countable then it is first countable so $X$ is maximal pseudocompact and the theorem is trivially true. If $X$ is uncountable then let $\kappa=|X|^{+}$and consider the space $Y=X \times A(\kappa)$ where $A(\kappa)$ is the one-point compactification of a discrete space of cardinality $\kappa$, i.e., $A(\kappa)=D \cup\{p\}$ where $|D|=\kappa$ and $p$ is the unique non-isolated point of $A(\kappa)$.

Observe first that $t(Y) \leq \omega$ by [1, Theorem 2.3.2]. If $E$ is a countable subset of $Y$ then $\bar{E}$ is contained in $X \times M$ where $M$ is a metrizable compact subspace of $A(\kappa)$. The space $X \times M$ is Fréchet-Urysohn by [1, Theorem 2.3.14] so every point of $\bar{E}$ is a limit of a sequence contained in $E$. This proves that $Y$ is a Fréchet-Urysohn space.

It is easy to find a family $\left\{S_{x}: x \in X\right\}$ of countably infinite disjoint subsets of the set $D$; let $T_{x}=\{x\} \times\left(S_{x} \cup\{p\}\right)$ for any $x \in X$. We claim that the set $K=\bigcup\left\{T_{x}: x \in X\right\}$ is closed in $Y$. Indeed, if $q \in Y \backslash K$ then $q=(x, d)$ for some $x \in X$ and $d \in D \backslash S_{x}$. The set $V=X \times\{d\}$ is an open neighbourhood of $q$ and $V \cap K$ contains at most one point of $K$ so $K$ is compact being closed in $Y$. The set $X \times\{p\}$ is a subspace of $K$ homeomorphic to $X$; if $u=(x, d) \in K$ then letting $r(u)=(x, p)$ we obtain a continuous retraction $r: K \rightarrow X \times\{p\}$. Therefore $X$ is homeomorphic to a retract of $K$.

If $x \in X$ then the point $(x, p)$ is in the closure of the set $\{x\} \times S_{x}$; besides, for any point $q=(x, d) \in\{x\} \times S_{x}$ the set $V=X \times\{d\}$ is an open neighbourhood of $q$ such that $V \cap K=\{(x, d)\}$ so every point of $K \backslash(X \times\{p\})$ is isolated in $K$. Since $K$ is a compact Fréchet-Urysohn space with a dense set of isolated points, it is maximal pseudocompact by $[3$, Theorem 2.4].

\section{Corollary 3.23.}

A continuous image of a compact maximal pseudocompact space need not be maximal pseudocompact.

Proof. The compact space $X$ from Example 3.15 is Fréchet-Urysohn but not maximal pseudocompact. Apply Theorem 3.22 to see that there exists a compact MP-space $K$ such that $X$ is a continuous image of $K$.

It was shown in [3] that there exist two scattered Fréchet-Urysohn compact spaces $X$ and $Y$ such that $X \times Y$ is not maximal pseudocompact. By [3, Theorem 2.16] both spaces $X$ and $Y$ are hereditarily maximal pseudocompact and hence they have the Preiss-Simon property by Corollary 3.13. Therefore the Preiss-Simon property is not productive even in compact spaces. However, we can prove a stronger result than Theorem 3.10 on products of hereditarily maximal pseudocompact spaces. 


\section{Theorem 3.24.}

If $X$ is a countably compact space with the Preiss-Simon property then the product $X \times Y$ has the Preiss-Simon property for any compact first countable space $Y$.

Proof. It is well known that the space $Z=X \times Y$ has to be countably compact. Fix any closed set $F \subset Z$ and a non-isolated point $z=(x, y) \in F$. Let $G=F \cap(\{x\} \times Y)$ and $U=F \backslash G$. If $z \notin \bar{U}$ then $V=F \backslash \bar{U} \in \tau(F)$ and $z \in V \subset G$. The space $G$ being first countable, it is easy to construct a sequence $\left\{U_{n}: n \in \omega\right\} \subset \tau^{*}(V)$ converging to $z$.

Now assume that $z \in \bar{U}$; the space $Z$ must be Fréchet-Urysohn by [1, Theorem 2.3.14] so we can find a faithfully indexed sequence $S=\left\{a_{n}: n \in \omega\right\} \subset U$ which converges to $z$. The set $G \backslash\{z\}$ is $\sigma$-compact by first countability of $Y$ so we can find a sequence $\left\{V_{n}: n \in \omega\right\} \subset \tau(F)$ such that $G \backslash\{z\} \subset \bigcup_{n \in \omega} V_{n}$ and $\bar{V}_{n} \cap S=\emptyset$ for any $n \in \omega$. Analogously, there exists a family $\left\{W_{n}: n \in \omega\right\} \subset \tau(F)$ such that $S \subset \bigcup_{n \in \omega} W_{n}$ and $\bar{W}_{n} \cap G=\emptyset$ for each $n \in \omega$. Exactly as in the proof of [6, Lemma 1.5.15] we can obtain disjoint sets $V, W \in \tau(F)$ such that $G \backslash\{z\} \subset V$ and $S \subset W$. Then $Q=\bar{W}$ is a closed subset of $F$ such that $Q \cap G=\{z\}$ and $z \in \bar{W}$.

Let $\pi: X \times Y \rightarrow X$ be the natural projection. The set $H=\pi(Q)$ is closed in $X$ and $x=\pi(z)$ is in the closure of the set $\pi(W) \subset H \backslash\{x\}$ so $x$ is a non-isolated point of $H$. By the Preiss-Simon property of $X$, we can find a sequence $\left\{E_{n}: n \in \omega\right\}$ of non-empty open subsets of $H \backslash\{x\}$ which converges to $x$. The set $B_{n}=\pi^{-1}\left(E_{n}\right) \cap Q$ is non-empty and open in $Q$; it is easy to see that the family $\mathcal{U}=\left\{B_{n}: n \in \omega\right\}$ is discrete in $Q \backslash \pi^{-1}(x)=Q \backslash\{z\}$. The set $Q$ being countably compact, the sequence $\mathcal{U}$ has the only accumulation point $z$, i.e., $\mathcal{U}$ converges to $z$. Therefore the sequence $\left\{B_{n} \cap W: n \in \omega\right\}$ consists of non-empty open subsets of $F$ and converges to $z$, i.e., we proved that $X \times Y$ has the Preiss-Simon property.

\section{Corollary 3.25 .}

If $X$ is a countably compact hereditarily maximal pseudocompact space then $X \times Y$ is hereditarily maximal pseudocompact for any compact first countable space $Y$.

\section{Open problems}

Although we have a lot of information on the properties and behaviour of maximal pseudocompactness, some natural questions about this concept are still open. We placed below a selection of some interesting questions we could not solve while working on this paper.

Question 1. Suppose that $X$ is maximal pseudocompact and $M$ is a second countable compact space. Must $X \times M$ be maximal pseudocompact?

Question 2. Suppose that $X$ is a compact MP-space and $M$ is a second countable compact space. Must $X \times M$ be maximal pseudocompact?

Question 3. Give an internal characterization of MP-spaces.

Question 4. Give an internal characterization of compact MP-spaces.

Question 5. Is it true that every compact space is embeddable in a compact MP-space?

Question 6. Is it true that every compact sequential space embeds in a compact MP-space?

Question 7. Observe that it follows from Proposition 3.1 that any separable MP-space has cardinality at most $2^{\omega}$; in particular, $\beta \omega$ is not an MP-space. But can $\beta \omega$ be embedded in a compact MP-space?

Question 8. Give an internal characterization of compact spaces representable as a continuous image of a compact MP-space.

Question 9. Is there a ZFC example of a compact MP-space of uncountable tightness? 
Question 10. Suppose that $X$ is a Preiss-Simon space and $f: X \rightarrow Y$ is a closed continuous onto map. Must $Y$ be a Preiss-Simon space?

\section{Acknowledgements}

The authors are very grateful to professor Alex Ravsky for pointing out that [9, Lemma 5.4] is applicable to prove that Question 3.10 has a positive answer for paracompact spaces.

Research supported by Promep 912011, Proyecto 12611768 (Mexico). The first and third authors are also supported by Fundação de Amparo a Pesquisa do Estado de São Paulo (Brasil). The second and third authors are also supported by CONACyT grant CB-2012-01-178103 (Mexico).

\section{References}

[1] Arhangel'skii A.V., Structure and classification of topological spaces and cardinal invariants, Russian Math. Surveys, 1978, 33(6), 33-96

[2] Arhangel'skii A.V., Relations among the invariants of topological groups and their subspaces, Russian Math. Surveys, 1980, 35(3), 1-23

[3] Alas O.T., Sanchis M., Wilson R.G., Maximal pseudocompact and maximal R-closed spaces, Houston J. Math., 2012, 38(4), 1355-1367

[4] Cameron D.E., A class of maximal topologies, Pacific J. Math., 1977, 70(1), 101-104

[5] Efimov B.A., Dyadic bicompacta, Trudy Moskov. Mat. Obshch., 1965, 14, 211-247 (in Russian)

[6] Engelking R., General Topology, IMPAN Monogr. Mat., 60, PWN, Warsaw, 1977

[7] Fabian M., Gâteaux Differentiability of Convex Functions and Topology, Canad. Math. Soc. Ser. Monogr. Adv. Texts, John Wiley \& Sons, New York, 1997

[8] Gillman L., Jerison M., Rings of Continuous Functions, The University Series in Higher Mathematics, Van Nostrand Princeton, 1960

[9] Gruenhage G., Generalized metric spaces, In: Handbook of Set-Theoretic Topology, North-Holland, Amsterdam, 1984, 423-501

[10] Knaster B., Urbanik K., Sur les espaces complets séparables de dimension 0, Fund. Math., 1953, 40, 194-202

[11] Preiss D., Simon P., A weakly pseudocompact subspace of Banach space is weakly compact, Comment. Math. Univ. Carolinae, 1974, 15(4), 603-609

[12] Porter J.R., Stephenson R.M. Jr., Woods R.G., Maximal feebly compact spaces, Topology Appl., 1993, 52(3), 203-219

[13] Porter J.R., Stephenson R.M. Jr., Woods R.G., Maximal pseudocompact spaces, Comment. Math. Univ. Carolinae, 1994, 35(1), 127-145

[14] Reznichenko E.A., Extension of functions defined on products of pseudocompact spaces and continuity of the inverse in pseudocompact groups, Topology Appl., 1994, 59(3), 233-244 